Notre Dame Journal of Formal Logic

Volume XI, Number 4, October 1970

\title{
THE DEPENDENCE OF A MEREOLOGICAL AXIOM
}

\author{
ROBERT E. CLAY
}

In this note we show that in the standard axiom system for mereology which follows, the reflexive axiom, $M 2$, is dependent on $M 3, D M, M 4$, and M5.

M1. $[A B]: A \varepsilon \mathrm{el}(B) . \supset . B \varepsilon B$.

M2. $[A]: A \varepsilon A . \supset . A \varepsilon \operatorname{el}(A)$.

M3. $[A B C]: A \varepsilon$ el $(B) . B \varepsilon \mathrm{el}(C) . \supset . A \varepsilon$ el $(C)$.

DM. $[A a] . \therefore A \varepsilon \mathrm{KI}(a) . \equiv: A \varepsilon A:[D]: D \varepsilon a . \supset . D \varepsilon \operatorname{el}(A):[D]:$

$D \varepsilon \operatorname{el}(A) . \supset .\left[{ }_{\exists} E F\right] . E \varepsilon a . F \varepsilon \operatorname{el}(D) . F \varepsilon \operatorname{el}(E)$.

M4. $[A a]: A \varepsilon a . \supset .[\exists B] . B \varepsilon \mathrm{KI}(a)$.

M5. $[A B a]: A \& \mathrm{KI}(a) . B \varepsilon \mathrm{KI}(a) . \supset . A \varepsilon B$.

P1. $\left.[A D]: A \varepsilon A . D \varepsilon \operatorname{el}(A) . \supset .{ }_{\exists} F\right] . F \varepsilon \operatorname{el}(D)$

$P F \quad[A D]: \operatorname{Hyp}(2) . \supset$.
$\left[{ }_{\exists} B\right]$.
3) $B \varepsilon \mathrm{KI}(A)$.
4) $A \varepsilon \operatorname{el}(B)$.
5) $D \varepsilon \operatorname{el}(B)$.
$\left[{ }_{\exists} F\right] . F \varepsilon$ el $(D)$

$[M 4,1]$

$[D M, 3,1]$

$[M 3,2,4]$

P2. $[A]: A \& A . \supset . A \varepsilon \mathrm{KI}(\mathrm{el}(A))$

$[D M, 3,5]$

$[D M, a / \mathrm{el}(A), E / D, P 1]$

P3. $\left.[A B D]: A \varepsilon A . B \varepsilon \mathrm{KI}(A) . D \varepsilon \mathrm{el}(B) . \supset .{ }_{\exists} E F\right] . E \varepsilon$ el $(A)$.

$F \varepsilon \mathrm{el}(D) . F \varepsilon \mathrm{el}(E)$

$P F \quad[A B D]: \operatorname{Hyp}(3) . \supset$.
4) $A \varepsilon \mathrm{el}(B)$.
$[D M, 2,1]$
$\left[{ }_{\exists} E F\right]$.
5) $E \varepsilon A$.
6) $F \varepsilon \mathrm{el}(D)$
7) $F \varepsilon \mathrm{el}(E)$.
$[D M, 2,3]$
8) $E=A$.
$[5,1]$
9) $F \varepsilon$ el $(A)$.
$[7,8]$
10) $F \varepsilon \mathrm{el}(B)$.
$[M 3,6,3]$ $\left[{ }_{\exists} G\right]$.
11) $G \in \mathrm{el}(F)$.
[DM, 2, 10]
12) $G \varepsilon \mathrm{el}(D)$.
$[M 3,11,6]$
$\left[{ }_{\exists} E F\right] . E \varepsilon \mathrm{el}(A) . F \varepsilon \mathrm{el}(D) . F \varepsilon \mathrm{el}(E)$
$[9,12,11]$ 
P4. $[A]: A \varepsilon A . \supset . A \varepsilon \operatorname{el}(A)$

PF $[A] \therefore A \varepsilon A . \supset$ :
$\left[{ }_{\exists} B\right]:$
2) $B \in \mathrm{KI}(A)$.
3) $A \varepsilon \operatorname{el}(B)$ :
4) $[D]: D \varepsilon \operatorname{el}(A) . \supset . D \varepsilon \mathrm{el}(B)$ :
5) $[D]: D \varepsilon \mathrm{el}(B) . \supset \cdot\left[{ }_{\exists} E F\right] . E \varepsilon$ el $(A) . F \varepsilon \mathrm{el}(D)$. $F \varepsilon \operatorname{el}(E)$ :
6) $B \varepsilon \operatorname{KI}(e l(A))$.
7) $A \varepsilon \operatorname{KI}(e l(A))$.
8) $A=B$ :
9) $A \varepsilon \operatorname{KI}(A)$.
$A \varepsilon \operatorname{el}(A)$

$$
\begin{array}{r}
{[M 4,1]} \\
{[D M, 2,1]} \\
{[M 3,3]} \\
{[P 3,1,2]} \\
{[D M, 2,4,5]} \\
{[P 2,1]} \\
{[M 5,6,7]} \\
{[2,8]} \\
{[D M, 9,1]}
\end{array}
$$

University of Notre Dame

Notre Dame, Indiana 